\title{
The DNL absorbing boundary condition: applications to wave problems
}

\author{
M.A. Storti *, J. D’Elía, R.P. Bonet Chaple, N.M. Nigro, S.R. Idelsohn \\ Centro Internacional de Métodos Numéricos en Ingeniería, CIMEC, INTEC (CONICET-UNL), Güemes 3450, 3000 Santa Fe, \\ Argentina \\ Received 1 September 1998
}

\begin{abstract}
A general methodology for developing absorbing boundary conditions is presented. For planar surfaces, it is based on a straightforward solution of the system of block difference equations that arise from partial discretization in the directions transversal to the artificial boundary followed by discretization on a constant step 1D grid in the direction normal to the boundary. This leads to an eigenvalue problem of the size of the number of degrees of freedom in the lateral discretization. The eigenvalues are classified as rightor left-going and the absorbing boundary condition consists in imposing a null value for the ingoing modes, leaving free the outgoing ones. Whereas the classification is straightforward for operators with definite sign, like the Laplace operator, a virtual dissipative mechanism has to be added in the mixed case, usually associated with wave propagation phenomena, like the Helmholtz equation. The main advantage of the method is that it can be implemented as a black-box routine, taking as input the coefficients of the linear system, obtained from standard discretization (FEM or FDM) packages and giving on output the absorption matrix. We present the application of the DNL methodology to typical wave problems, like Helmholtz equations and potential flow with free surface (the ship wave resistance and sea-keeping problems). (c) 2000 Elsevier Science S.A. All rights reserved.
\end{abstract}

Keywords: Potential flow; Finite element method; Wave resistance; Absorbing boundary condition; Free surface flow; Partial discretization

\section{Introduction}

When solving elliptic problems in unbounded domains with numerical methods, like the Finite Element Method (FEM) or Finite Difference Method (FDM) one faces the problem of truncating the domain at a certain artificial boundary. For positive definite operators, like the Laplace operator or the elasticity equations, imposing null Dirichlet conditions at an artificial boundary located far enough from the region of interest is enough, in the sense that pushing this boundary to infinity converges to the unbounded solution (i.e. the Cauchy problem). Essentially the same thing happens for Neumann or mixed boundary conditions. The convergence to the unbounded solution may depend on space dimension, and on the order of the perturbation (i.e. if it can be approximated by a single pole, dipole, or higher order term), and some numerical techniques, like coupling with an external Boundary Integral solution or infinite elements, have been developed in order to minimize the computational effort.

In contrast, for wave-like problems like the Helmholtz equation, the situation is much more complex. The process of pushing the boundary to infinity may be not convergent at all unless an appropriate boundary condition is imposed in the artificial boundary. For instance in $2 \mathrm{D}$, the solution is known to

\footnotetext{
${ }^{*}$ Corresponding author. Tel.: +54-342-455-9175; fax: +54-342-455-0944.

E-mail address: mstorti@minerva.unl.edu.ar (M.A. Storti).
} 
decay to infinity at least as $1 / \sqrt{r}(1 / r$ in $3 \mathrm{D})$ due to dispersion of the radiating energy and one could try to impose homogeneous Dirichlet or Neumann boundary conditions at the artificial boundary. But doing so, the artificial boundary acts like a closed cavity which can enter in resonance modes, as the artificial boundary recedes to infinity. Then the potential at a given point passes through infinite values at least twice each time the radius of the artificial boundary is receded one wave-length, so that this is clearly not a convergent process. A key-point in the understanding of this lack of convergence is that its origin is in the absence of physical dissipation. Effectively, as the medium is non-dissipative, the energy flux irradiated by the body arrives to the artificial boundary and is reflected back to the center of the domain. When the gain of the closed loop is infinity a resonance of the cavity occurs.

A small amount of dissipation regularizes the problem. For the Helmholtz equation, dissipation can be added with a small positive imaginary part i $\delta$ to the refraction index of the medium. Let $\phi_{\delta, R}$ be the solution for a given viscosity parameter $\delta \rightarrow 0^{+}$and $R \rightarrow \infty$ the radius of the artificial boundary. Then for $\delta>0$ we can consider the unbounded viscous solution

$$
\phi_{\delta, \infty}=\lim _{R \rightarrow \infty} \phi_{\delta, R}
$$

This solution is independent of the boundary conditions imposed at infinity. Now, we can safely take the limit of negligible viscosity, and we arrive to the unbounded inviscid solution

\section{$=\lim _{\delta \rightarrow 0^{+}}\left\{\lim _{R \rightarrow \infty} \phi_{\delta, R}\right\}$}

A key point is that the order in taking the limits does matter. A closer analysis of the unbounded inviscid solution shows that only outgoing components are present in the far-field, where outgoing means here that the group velocity of the wave points from the domain interior to infinity. Thus, absorbing boundary

conylitions may be constructect

The sense of propagation may

computing the group velocity

direction in which the compos

e simplest absorbing

rormally to the boundary and then a
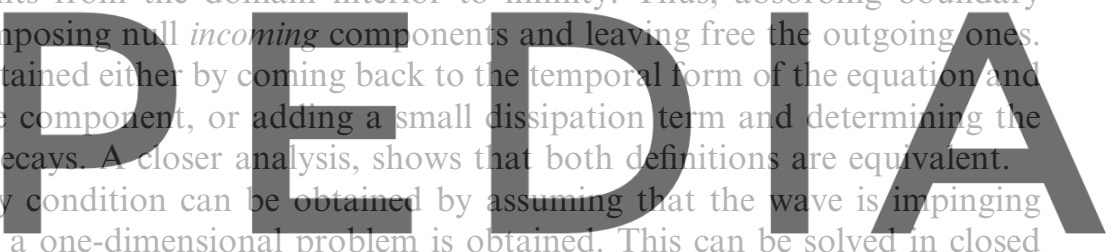

form, and the first order absorbing boundary condition is obtained. Here first order, means that the re-

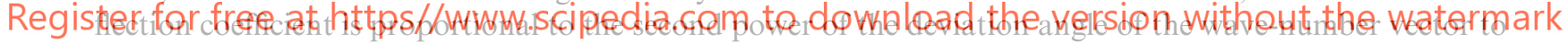

normal incidence. Higher order absorbing boundary conditions, i.e. those ones whose reflection coefficients are proportional to higher powers of the deviation angle, have been proposed, based on different kinds of expansions in the deviation angle. On the other hand, exact absorbing boundary conditions have been also developed. They can be put at a finite distance and there is no need of pushing it to infinity in order to converge to the unbounded inviscid solution. In general they are based on matching the internal solution with a representation of the external field in a form of series (the DtN method, see [8-10]), a Boundary Element solution [19], or infinite elements [2,5].

In this work we will present an exact boundary condition derived in a purely algebraic form. It is based on the exact solution of the system of discrete difference equations (or the system of ODE's obtained by semidiscretization in the transversal directions). Standard operational techniques lead to a quadratic eigenvalue problem (for second order elliptic equations) which is solved with standard routines. Eigenvalues are classified as left- or right-going depending on their absolute value, and the far-field general form for the solution is allowed to include only outgoing (i.e. right-going for a boundary at the right and left-going for a boundary at the left). Algebraic manipulation of this general form leads to the wanted absorbing boundary condition.

\section{General form of the discrete equations}

We start with the simplest case, that is the Helmholtz equation with constant refraction index in a cylinder formed by the extrusion of a certain 2D section $\Sigma_{y z}$ in the $y z$ plane with boundary $\Gamma_{y z}$, in the $x$ direction 


$$
\Omega_{L}=\left\{x, y, z /-L<x<L \quad(y, z) \in \sum_{y z}\right\} .
$$

Dirichlet boundary conditions are imposed at the boundary $\Sigma$ composed of all the points $(x, y, z)$ such that $(y, z)$ is in $\Gamma_{y z}$ (see Fig. 1). As mentioned before, we add a small dissipation term, and impose homogeneous Dirichlet boundary conditions at some section $x= \pm L$. The governing equations are, then

$$
\begin{aligned}
& \Delta \phi_{\delta, L}+k^{2}(1+\mathrm{i} \delta) \phi_{\delta, L}=f \quad \text { in } \Omega_{L}, \\
& \phi_{\delta, L}=0 \quad \text { at } \Sigma \quad \text { and } \quad x= \pm L .
\end{aligned}
$$

We assume that $f$ has compact support, i.e.

$$
f=0 \text { for }|x|>x_{\max } .
$$

We proceed to a FEM discretization in two steps. First we semi-discretize in the $y z$ variables and secondly a 1D discretization with constant step size $\Delta x$ in $x$. The first step gives a coupled system of ODE's of the form

$$
\mathbf{M} \mathbf{u}_{x x}-\mathbf{K u}+k^{2}(1+\mathrm{i} \delta) \mathbf{M u}=\mathbf{f}(x),
$$

where

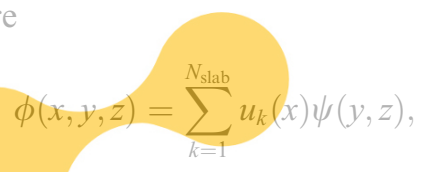

is the FEM approximation in the section, and
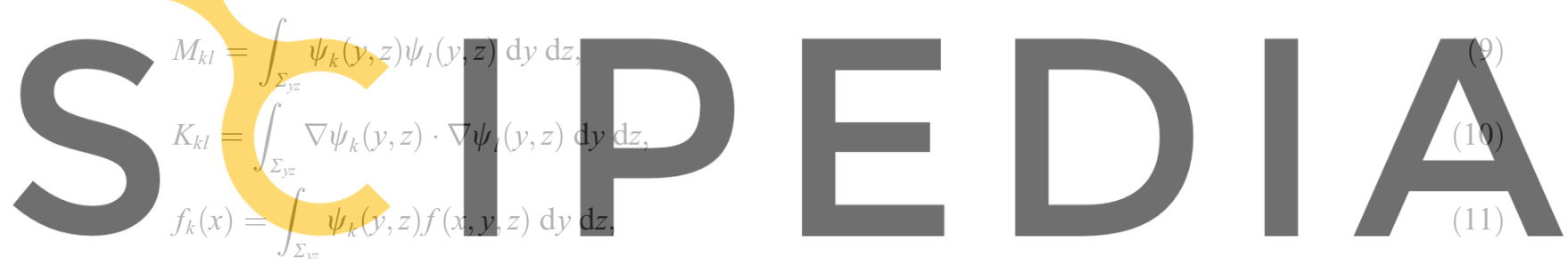

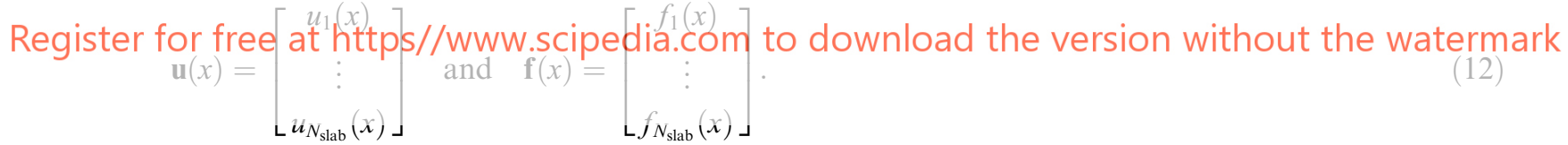

Note that $\mathbf{M}$ and $\mathbf{K}$ are the typical FEM mass and stiffness matrices for the section, and then they are $s p d$ (symmetric and positive definite).

1D FEM discretization in $x$ of (7) with linear elements of constant size $\Delta x=L / N$ is obtained by approximating

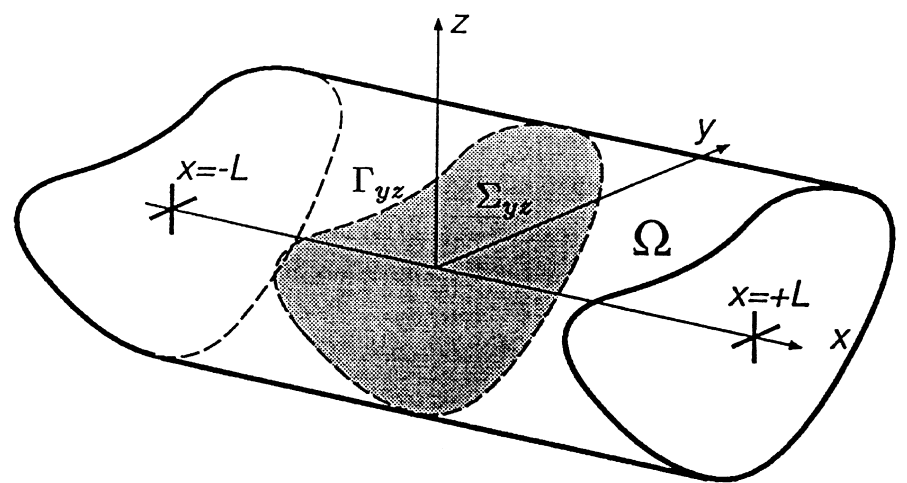

Fig. 1. Cylinder shaped domain. 


$$
\mathbf{u}(x)=\sum_{l=-N+1}^{N-1} \mathbf{u}_{l} w_{l}(x),
$$

where $w_{l}$ are the typical hat interpolation functions, and gives

$$
\mathbf{A} \mathbf{u}_{k+1}+\mathbf{B} \mathbf{u}_{k}+\mathbf{C} \mathbf{u}_{k-1}=\mathbf{f}_{k}^{\prime}, \quad \text { for } k=-N+1, \ldots, N-1,
$$

where

$$
\begin{aligned}
& \mathbf{A}=\mathbf{C}=\mathbf{M}+\frac{1}{6}\left(\bar{k}^{2} \mathbf{M}-\mathbf{K}\right) \Delta x^{2} \\
& \mathbf{B}=-2\left[\mathbf{M}-\left(\Delta x^{2} / 3\right)\left(\bar{k}^{2} \mathbf{M}-\mathbf{K}\right)\right]
\end{aligned}
$$

and

$$
\begin{aligned}
& \mathbf{f}_{j}^{\prime}=\left(\Delta x^{2} / 6\right)\left(\mathbf{f}_{j+1}+4 \mathbf{f}_{j}+\mathbf{f}_{j-1}\right), \\
& \bar{k}^{2}=k^{2}(1+\mathrm{i} \delta) .
\end{aligned}
$$

Here $\mathbf{u}_{k}$ stands for the vector of nodal potentials at the nodes on layer $k$. The Dirichlet boundary conditions at $x= \pm L$ are imposed by assuming $\mathbf{u}_{ \pm N}=\mathbf{0}$. This same equations could be obtained by performing a full discretization and afterwards a block splitting in blocks of size $N_{\text {slab }} \times N_{\text {slab }}$. The system matrix results to be block tri-diagonal, and the block coefficient matrices are constant (i.e. not depending on $j$ ) and given by the A, B, C matrices given before

3. Solution for the discrete
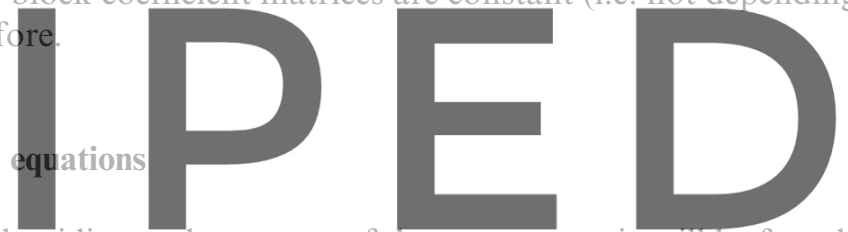

It is clear that such block-tridiagonal structure of the system matrix will be found whenever the operator is linear and homogeneous (i.e. not depending on $x$ ), from positive definite operators like Laplace or the

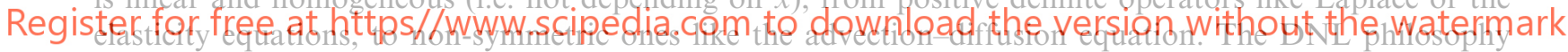

exploits the constant coefficient block matrix system of difference (14) in order to find an explicit form of the solution. Once this solution is found, it is used in order to develop absorbing boundary conditions.

Let us look for solutions to the homogeneous version of (14) in the form

$$
\mathbf{u}_{k}=\mu^{k} \hat{\mathbf{u}} .
$$

Replacing in (14) results in the characteristic equation

$$
\left(\mu^{2} \mathbf{A}+\mu \mathbf{B}+\mathbf{C}\right) \hat{\mathbf{u}}=0 .
$$

This is a quadratic eigenvalue problem. By a simple transformation it can be transformed to a linear eigenvalue problem. Let us define

$$
\mathbf{U}=\left[\begin{array}{c}
\mu \hat{\mathbf{u}} \\
\hat{\mathbf{u}}
\end{array}\right] .
$$

Then the quadratic eigenvalue problem is equivalent to the following linear one

$$
\left[\begin{array}{cc}
-\mathbf{A}^{-1} \mathbf{B} & -\mathbf{A}^{-1} \mathbf{C} \\
\mathbf{I} & \mathbf{0}
\end{array}\right] \mathbf{U}=\mu \mathbf{U}
$$

For symmetric operators, like the Helmholtz or Laplace operators, it happens that $\mathbf{C}=\mathbf{A}$ and then the problem can be reduced to a generalized eigenvalue problem of size $N_{\text {slab }} \times N_{\text {slab }}$ by the transformation

$$
\eta=(\mu+1 / \mu) / 2 .
$$


Effectively, replacing (23) in (20) we arrive to

$$
\mathbf{B} \hat{\mathbf{u}}=-2 \eta \mathbf{A} \hat{\mathbf{u}} .
$$

This is computationally more efficient than solving (22). Once the $\eta_{k}$ are obtained, the $\mu_{k}$ can be obtained by solving the quadratic (23). Note that each $\eta$ gives raise to two $\mu$ eigenvalues

$$
\mu_{k}^{ \pm}=-\eta_{k} \pm \sqrt{\eta_{k}^{2}-1}
$$

It is easy to show that both roots satisfy $\mu_{k}^{+} \mu_{k}^{-}=1$, so that there are two possibilities.

- Viscous case: $\left|\mu_{k}^{+}\right|<1<\left|\mu_{k}^{-}\right|$or $\left|\mu_{k}^{-}\right|<1<\left|\mu_{k}^{+}\right|$.

- Inviscid case: $\left|\mu_{k}^{ \pm}\right|=1$.

In addition, in the viscous case we classify the eigenvalue which has $|\mu|<1$ as right-going, and that one which is $|\mu|>1$ as left-going. The reason for this is that the general solution for $x \rightarrow+\infty$ should only include terms $\propto \mu^{k}$ with $|\mu|<1$, so that we should impose those components with $|\mu|>1$ to zero on right boundaries. Recall now that the general rule for absorbing boundary conditions is to impose the incoming components and to leave free the outgoing ones, so that this implies that $|\mu|>1$ are incoming (i.e., leftgoing), and $|\mu|<1$ are outgoing (i.e., right-going). Applying the reasoning to a left boundary gives the same result.

For the non-dissipative Helmholtz equations $(\delta=0)$ it happens that there is a set of inviscid eigenvalues that correspond physically to propagating modes, and viscous eigenvalues which correspond to evanescent modes. In what follows we will extend the concept of right-going and left-going to this case. We can do it in two equivalent forms. First, we can add a dissipative term and see in what direction move this inviscid eigenvalues. This has to be done only for the sake of classification, afterwards we can let $\delta \rightarrow 0$ and recover the non-dissipative $\delta=0$ case. The other possibility is to come back to the temporal equation and compute

\section{the group velocities, this will}

fact equivalent in a very ge

Let us find first the eige

singular) and $\Lambda_{\beta}=\operatorname{diag}\{f$

value problem

$\mathbb{K W}=\mathbf{M W} \Lambda_{\beta}$.
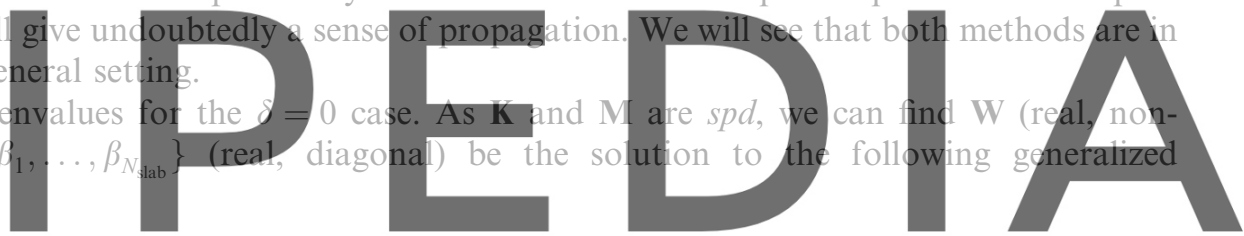

(26)

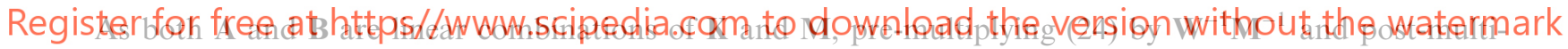

plying by W gives a decoupled system of equations which defines the $\eta$ eigenvalues in terms of the $\beta$ eigenvalues

$$
\eta=\frac{1-\left(\Delta x^{2} / 3\right)\left[k^{2}(1+\mathrm{i} \delta)-\beta\right]}{1+\left(\Delta x^{2} / 6\right)\left[k^{2}(1+\mathrm{i} \delta)-\beta\right]}
$$

As all the $\beta_{k}$ 's are real we see from (27) that the $\eta_{k}$ 's are also real for $\delta=0$. Now, from (25) we can see that for real $\eta$ the pair of eigenvalues are viscous if $|\eta|>1$ and inviscid if $|\eta| \leqslant 1$. Consider now the inviscid eigenvalues $\mu_{k}^{ \pm}$as a function of $\delta$, i.e. $\mu_{k}^{ \pm}(\delta)$, we will assume a regular expansion to first order in $\delta$ of the form

$$
\mu_{k}^{ \pm}(\delta)=\mu_{k}^{ \pm}(0)+\left.\frac{\mathrm{d} \mu}{\mathrm{d} \delta}\right|_{\mu_{ \pm(0)}^{k}} \delta+\mathrm{O}\left(\delta^{2}\right),
$$

and compute the derivative by the chain rule

$$
\frac{\mathrm{d} \mu}{\mathrm{d} \delta}=\frac{\mathrm{d} \mu}{\mathrm{d} \eta} \frac{\mathrm{d} \eta}{\mathrm{d} \delta}
$$

The factor $(\mathrm{d} \eta / \mathrm{d} \delta)$ can be computed straightforwardly from (27) and gives

$$
\left.\frac{\mathrm{d} \eta}{\mathrm{d} \delta}\right|_{\delta=0}=-\mathrm{i} \frac{\Delta x^{2} / 2}{\left[1+\left(\Delta x^{2} / 6\right)\left(k^{2}-\beta\right)\right]^{2}}<0 .
$$




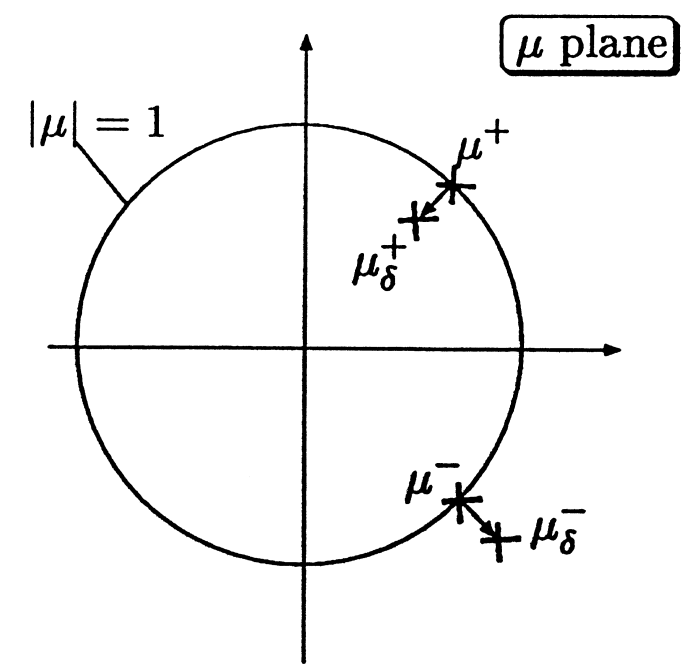

Fig. 2. Perturbation analysis for the inviscid eigenvalues.

\section{The factor $(\mathrm{d} \mu / \mathrm{d} \eta)$ can be computed from (23) and gives}

$$
\left(\frac{\mathrm{d} \mu}{\mathrm{d} \eta}\right)^{ \pm}=-\mu /(\mu+\eta)=-\mu /\left( \pm \mathrm{i} \sqrt{1-\eta^{2}}\right)
$$

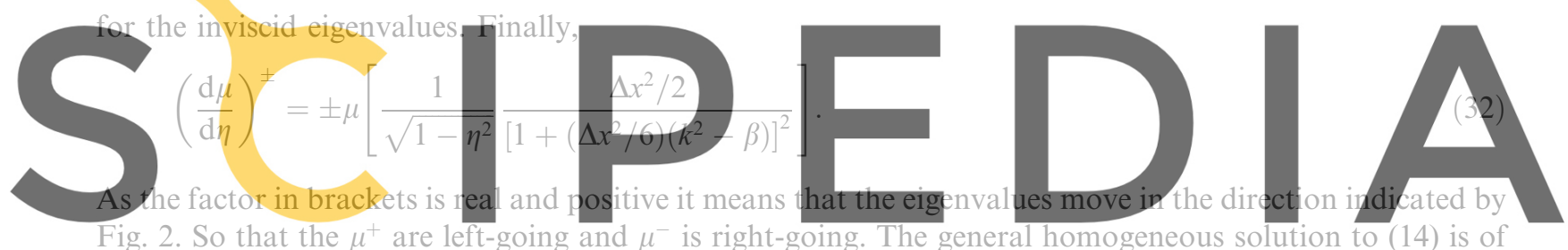

Register for free at https//www.scipedia.com to download the version without the watermark

$$
\mathbf{u}_{H, j}=\sum_{k=1}^{N_{\text {slab }}}\left[a_{k}^{\mathrm{R}}\left(\mu_{k}^{\mathrm{R}}\right)^{j}+a_{k}^{\mathrm{L}}\left(\mu_{k}^{\mathrm{L}}\right)^{j}\right] \mathbf{w}_{k} \cdot
$$

\section{Absorbing boundary conditions}

The development of absorbing boundary conditions is now straightforward. Consider first a right boundary. Due to (6), it results that the equation is homogeneous for $j>x_{\max } / \Delta x$ and then the general solution (33) applies. But, imposing the homogeneous boundary condition at $x=L$ and letting $L \rightarrow \infty$ shows that only the decaying (i.e. the right-going components) may exist.

$$
\mathbf{u}_{j}=\sum_{k=1}^{N_{\text {slab }}} a_{k}^{\mathrm{R}}\left(\mu_{k}^{\mathrm{R}}\right)^{j} \mathbf{w}_{k},
$$

then

$$
\begin{aligned}
\mathbf{u}_{k+1} & =\sum_{k=1}^{N_{\text {slab }}} a_{k}^{\mathrm{R}}\left(\mu_{k}^{\mathrm{R}}\right)^{j} \mu_{k}^{\mathrm{R}} \mathbf{w}_{k}, \\
& =\mathbf{W} \Lambda^{\mathrm{R}} \mathbf{W}^{-1} \mathbf{u}_{k}, \\
& =\mathbf{G}^{\mathrm{R}} \mathbf{u}_{k} .
\end{aligned}
$$


We can think at $\mathbf{G}^{\mathrm{R}}$ as the right propagator. Then

$$
\mathbf{u}_{N}=\mathbf{G}^{\mathrm{R}} \mathbf{u}_{N-1},
$$

is the absorbing boundary condition. Similarly, the left propagator is defined as

$$
\mathbf{G}^{\mathrm{L}}=\mathbf{W}\left(\Lambda^{\mathrm{L}}\right)^{-1} \mathbf{W}^{-1}
$$

and

$$
\mathbf{u}_{-N}=\mathbf{G}^{\mathrm{L}} \mathbf{u}_{-N+1},
$$

is an absorbing boundary condition at a left boundary. For symmetric operators $\left(\Lambda^{\mathrm{L}}\right)^{-1}=\Lambda^{\mathrm{R}}$ and then $\mathbf{G}^{\mathrm{L}}=\mathbf{G}^{\mathrm{R}}$.

\section{General relation between sense of propagation and sense of decay}

Consider a dispersive homogeneous medium and a non-dissipative branch of eigenvalues $\omega(k)$ so that waves in this branch are proportional (spatially and temporally) to $\mathrm{e}^{\mathrm{i}(\mathrm{kx}-\omega(\mathrm{k}) \mathrm{t})}$ for a given wave-number $k$. Suppose that we add a dissipative mechanism controlled by a small parameter $\delta$, such that the perturbed dispersion relation is $\bar{\omega}(k, \delta)$. As the term is dissipative, it means that, for $\delta>0$ we should have $\mathrm{e}^{\mathrm{i}(\mathrm{kx}-\bar{\omega}(\mathrm{k}, \delta) \mathrm{t})}$ decaying exponentially in time so that $\operatorname{Im}\{\bar{\omega}(k, \delta)\}>0$ for $\delta>0$. That means that
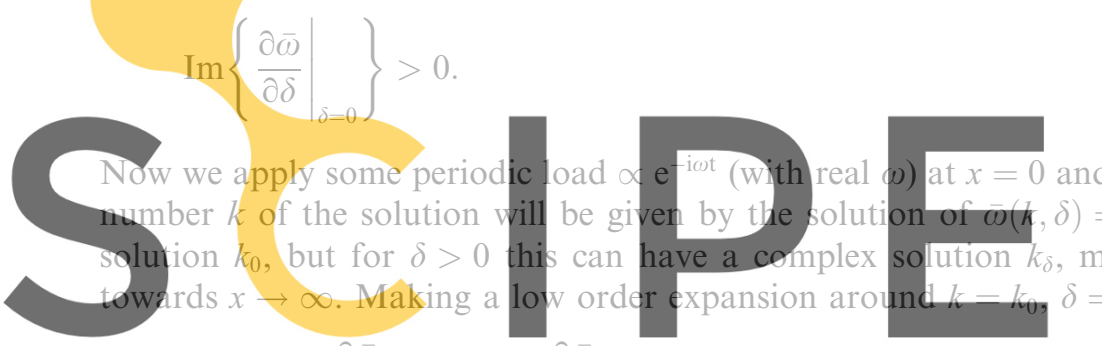

$\bar{\omega}\left(k_{0}, 0\right)+\frac{\partial \bar{\omega}}{\partial t}\left(k_{\delta}-k_{0}\right)+\frac{\partial \bar{\omega}}{\partial \delta} \delta=\omega$,

Register for free at https//www.scipedia.com to download the version without the watermark

but $\bar{\omega}\left(k_{0}, 0\right)=\omega$ so that

$$
\left(k_{\delta}-k_{0}\right)=-\frac{(\partial \bar{\omega} / \partial \delta)}{(\partial \bar{\omega} / \partial k)} \delta .
$$

Now $(\partial \bar{\omega} / \partial k)=v_{G}$ is the well known group velocity and is real since $\omega$ is real for $k$ real, and then by (41)

$$
\operatorname{sgn}\left(\operatorname{Im}\left\{k_{\delta}\right\}\right)=\operatorname{sgn}\left(\operatorname{Im}\left\{k_{\delta}-k_{0}\right\}\right)=\operatorname{sgn} v_{G},
$$

where $\operatorname{sgn}()$ denotes the sign function. Then, $\mathrm{e}^{\mathrm{i} \mathrm{k}_{\delta} \mathrm{x}}$ will decay towards $x \rightarrow+\infty$ for $v_{G}>0$ and towards $x \rightarrow-\infty$ for $v_{G}<0$. Note that here we assumed that $\bar{\omega}$ is analytic near $k_{0}$, since $v_{G}$ is the derivative of $\bar{\omega}$ along the real axis, whereas the derivative appearing in (43) coming from the Taylor expansion in (42) is along a non-real direction.

\section{Implementation details and generalizations}

\subsection{Inhomogeneities, polar (or spherical) coordinates and tail problems}

The DNL absorbing boundary condition results to be an exact condition in the sense that the numerical results converge to the exact solution even if the position of the artificial boundary is not receded to infinity, and resembles in this respect the DtN condition proposed in [8-10]. The main advantage of the DNL approach is that it can be implemented as a black-box routine working on the layer matrices $\mathbf{A}, \mathbf{B}$ and $\mathbf{C}$ 
that are computed with a standard finite element package. Even if the method has been described here for homogeneous operators on 1D structured meshes, it can (and in fact, its real possibilities of application to the real world depends on this) be extended to more general situations.

First of all, the operator needs to be homogeneous only near the region, where the general solution (33) is needed. For instance, for absorbing boundary conditions this is only needed near the artificial boundary, so that one can have a non-homogeneous operator in the interior region and a non-structured grid, and a small number of structured layers on a region of homogeneous operator at the artificial boundary.

If the block coefficients depend on position (i.e. $\mathbf{A}=\mathbf{A}^{j}$ ), but in such a way that they merge smoothly in $\mathbf{A}^{\infty}$ as $j \rightarrow \infty$, then some error is introduced when cutting the domain at certain $j=N$. These are called tail problems. For instance a sea bottom that slowly approximates to the limit depth or a base flow for the wave-resistance problem. 2D or 3D problems in polar or spherical geometry can be considered in the same way if we map the exterior annular region to a rectangular one. Some terms proportional to powers of $1 / r$ appear, and they can be seen as inhomogeneities. The strategy in this case is to create a structured condensing layer covering $J_{1} \leqslant j \leqslant J_{2}$. The planar DNL boundary condition is imposed at the exterior layer $\left(j=J_{2}\right)$ and then this condition is condensed back to $j=J_{1}$. This condensation process involves some computational effort, but with an appropriate eigen-decomposition the computational effort scales as $\sim N_{\text {slab }}\left(J_{2}-J_{1}\right)$ denotes the number of nodes in the condensing layer, and the additional requirement in core memory is negligible. In this way, the planar boundary condition can be effectively imposed at external layers which are located several wave-lengths from the region of interest. In addition, convergence to the exact absorbing boundary condition can be improved by applying a Gaussian filter in the condensation region, this is described in detail by Bonet et al. [3].

6.2. The virtual dissipative operator

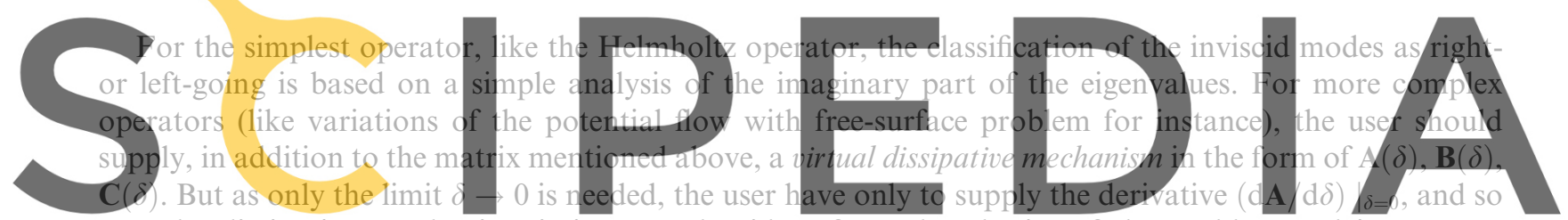

on. The dissipation mechanism is in general evident from the physics of the problem and it may not

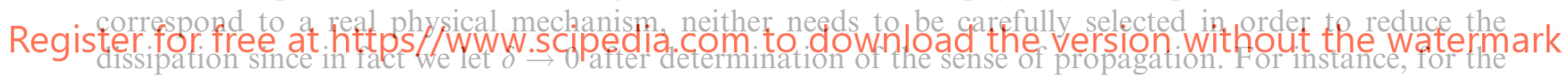
wave-resistance problem it is well known that a term proportional to $\phi_{x x x}$ or $-\phi_{x x x x}$ are dissipative. In practice the first results to be over-diffusive whereas the second is broadiy used in the family of Dawson codes. But for the sake of the determination of the sense of propagation described here, both are acceptable and give the same results.

\section{Numerical examples}

\subsection{Berkhoff (Helmholtz) equation}

The Berkhoff (see [1]) equation models the propagation of gravity waves in a varying depth bathymetry. Assuming a certain variation of the velocity profile in the vertical direction it results in a Helmholtz-like equation with a varying refraction index, so that the application of the DNL method described here is straightforward. We consider here the parabolic shoal problem that has been extensively studied theoretically and experimentally (see [11]). The geometry (see Fig. 3) consists in a parabolic variation of the bottom in the form $h(x, y)=\min \left\{h_{1}, h_{2}+\left(h_{1}-h_{2}\right)(r / R)^{2}\right\}$, where $r$ is the distance to the center of the shoal, located at the origin, $h_{1,2}=0.15$ and $0.05 \mathrm{~m}, R=0.8 \mathrm{~m}$ and the period of the plane inciding wave is $T=0.511 \mathrm{~s}$. (The wave-lengths computed from the dispersion relation are $\lambda_{1,2}=0.31$ and $0.4 \mathrm{~m}$.) So that, roughly, five wave-lengths enter in the diameter of the shoal. In the limit of very high frequency the rays are focused somewhere near point $A=(R, 0)$ at the exit of the shoal, resulting in a high concentration of energy which predicts high wave amplitudes. In Fig. 4 we see the wave profiles at sections passing through the focus, 


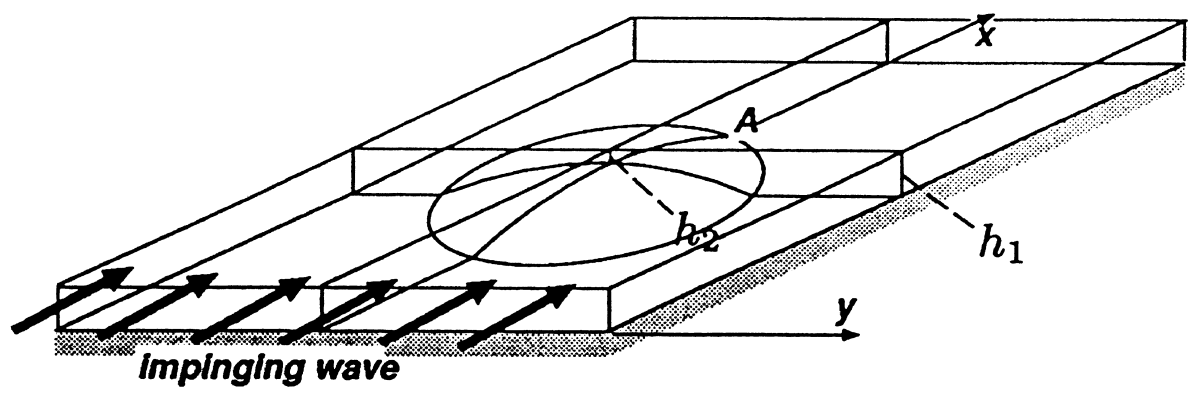

Fig. 3. Parabolic shoal: geometrical description.

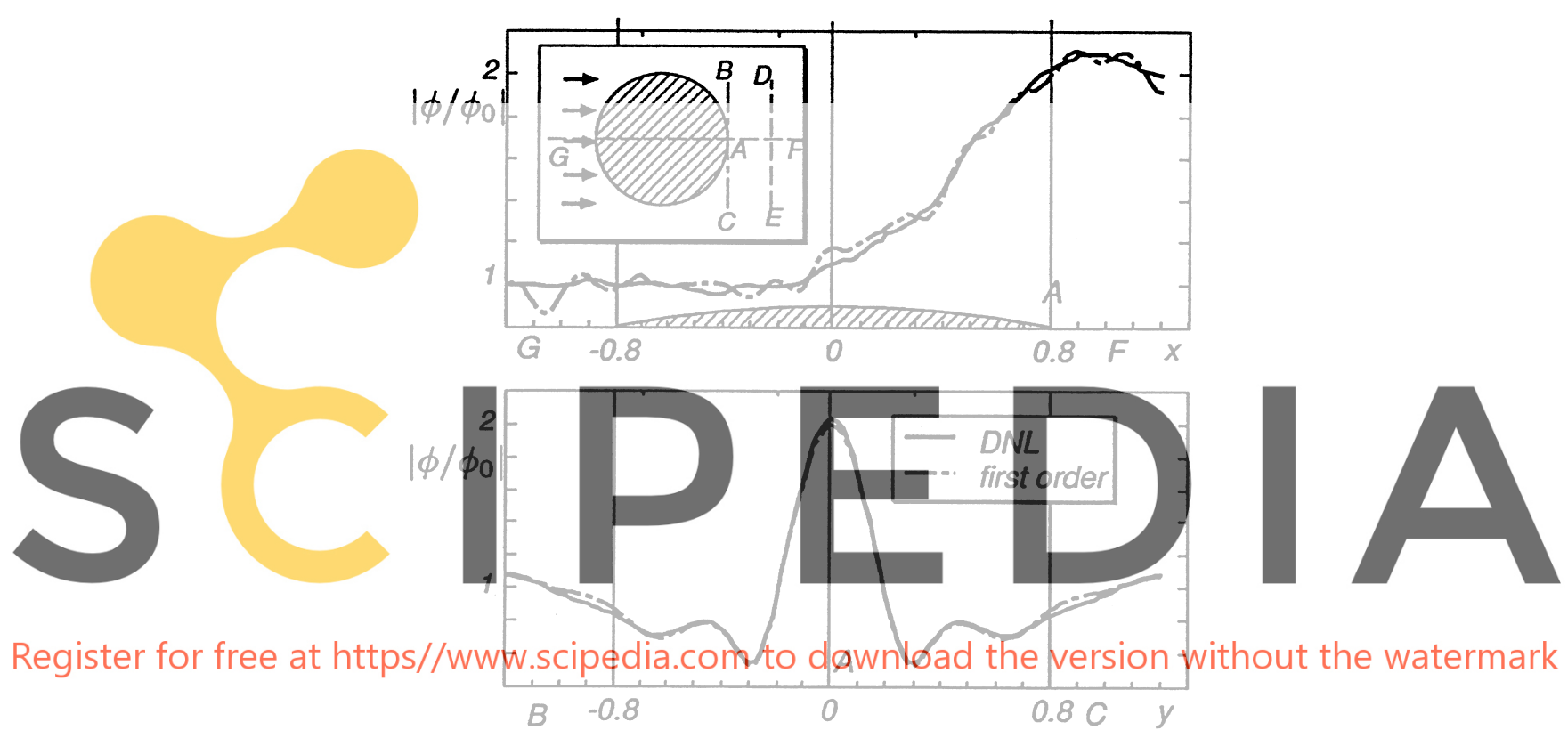

Fig. 4. Parabolic shoal: wave amplitude profiles at several sections passing through the focus.

compared with those obtained with the first order absorbing boundary condition. The outlet boundary condition (marked as $D E$ in the figure) is located at a distance $R / 2$ of the circle. For the first order results we see a series of oscillations typical of reflections in the longitudinal direction that are absent in the results obtained with the DNL. Finally, in Fig. 5 we see the elevation view of the wave amplitude on the region over the shoal.

\subsection{Ship wave resistance problems}

When a body moves near the free surface of a fluid, a pattern of trailing gravity waves is formed. The energy spent in building this pattern comes from the work done by the body against the wave resistance. Numerical modeling of this problem is a matter of high interest for ship design, and marine engineering (see $[6,18,12,15])$. As a first approximation, the wave resistance can be computed with a potential model, whereas for the viscous drag it can be assumed that the position of the surface is held fixed at the reference hydrostatic position, i.e. a plane. This is, basically, the Froude hypotheses [18].

We concentrate here in the computation of the flow field and wave resistance for a body in steady motion, by means of a potential model for the fluid and a linearized free surface boundary condition. This is 


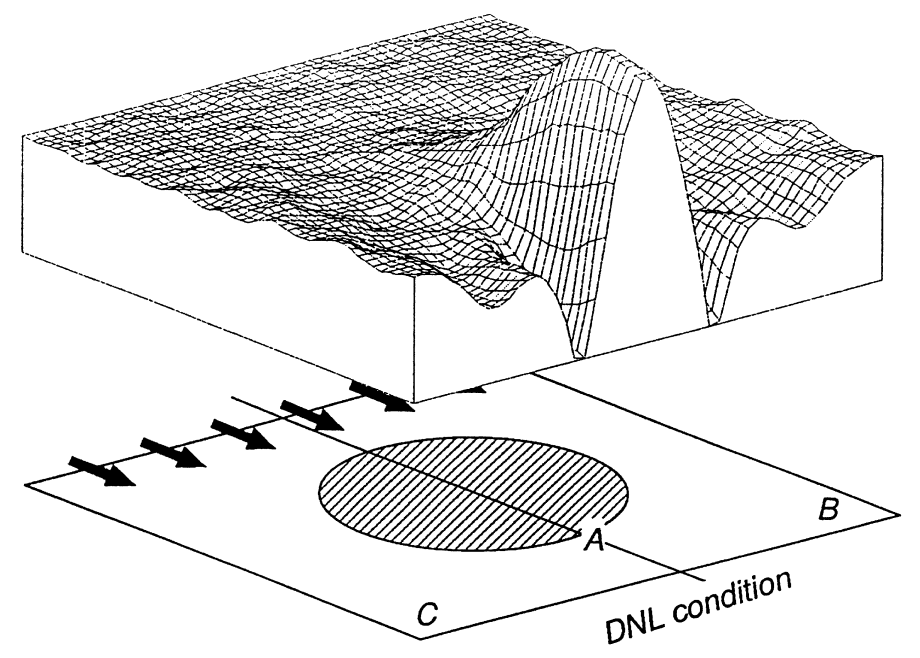

Fig. 5. Parabolic shoal: 3D view of wave amplitude.

the basis for most ship design codes in industry. The governing equations are the Laplace equation with slip boundary conditions on the hull and channel walls, inlet/outlet conditions at the corresponding planes and the free surface boundary condition. The free surface boundary condition amounts to a Neumann boundary condition with a source term proportional to the streamlined second derivative of the potential. However, the problem as stat ordinate inversion $(x \rightarrow-x)$, propagating downstream. To kind of absorbing boundary can be shown that the addition
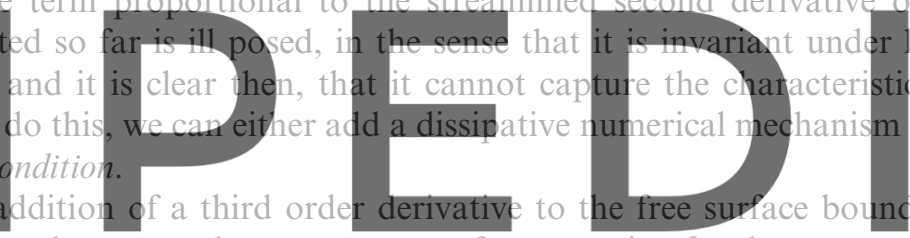
f the potential.
ongitudinal co-
trailing waves
or impose sone

adds a dissipative mechanism and captures the correct sense of propagation for the wave pattern [6]. This is Register falent to use a non-centered discretization scheme for the second order operator and falls among the

stream of the body. If the viscosity parameter is too low, the trailing waves arrive to the downstream boundary, are refiected in the upstream direction and poliute the solution. If it is too high, the trailing waves are damped and incorrect values of the drag are obtained. Extending the mesh in the downstream direction allows the use of a lower viscosity parameter, since the waves are damped in a larger distance, but increases the computational cost (core memory). Numerical experiences show that this third order streamline viscosity term is too dissipative and the meshes should be extended downstream too much. Dawson then proposed a method, where the fifth order derivative is used instead, with a very particular finite difference discretization. The fact is astonishing that standard discretization of the same operator does not work, neither do higher order operators (say seventh order). As a result, most today codes are still using some kind of variant of the Dawson scheme. However, this very particular viscosity term is hard to extend to general boundary fitted meshes, not mentioning to unstructured computational methods like finite elements. It is by this cause that most codes are based on a highly structured panel formulation.

Another possibility that is investigated here is to use an absorbing boundary condition in the downstream boundary. If such a numerical device could be found, then there is no need to add a numerical viscosity term, since the trailing waves are not reflected upstream, and a usual centered scheme can be used for the free surface boundary term. As a bonus, if such a centered scheme could be used, then the trailing waves would not dampen and the drag could be computed in terms of the momentum flow through a plane arbitrarily located downstream of the body. Broeze and Romate [4] developed an absorbing boundary condition for potential flow with a panel method but in the context of following a temporal evolution of the free surface problem and Lenoir and Tounsi [13] treated the sea-keeping problem, which is closer to the Helmholtz like equation than the wave-resistance problem. 
Application of the DNL methodology to the wave resistance problem is rather straightforward, but has a particular feature with respect to the sense of propagation of the components. (Full details can be found in $[16,17,7]$. Taking a computational domain in the form of a channel with rectangular section (for simplicity) with $N_{z}$ nodes in the draft (vertical) direction and $N_{y}$ in the beam (transversal) one it results that there are $2\left(N_{z}-1\right) N_{y}$ viscid eigenvalues and $2 N_{y}$ inviscid ones. Note that the number of inviscid modes coincides with twice the number of nodes on the surface, which is a direct physical consequence that these modes are associated with surface propagating waves. Now, the addition of $\phi_{x x x}$ or $-\phi_{x x x x x}$ as the virtual dissipative mechanism reveals that all the inviscid modes propagate in the downstream direction. Note that this coincides with the fact that the generated waves should propagate downstream. This poses a problem from the computational point of view, since a number of boundary conditions have to be moved from the downstream exit plane to the upstream inlet plane.

Several numerical results will be shown in 2D and 3D situations. Fig. 6 shows the wave resistance coefficient for a dipole submerged at a depth $f$. Recall that a dipole is equivalent to a very small cylinder in a potential flow, and the results are presented in terms of the diameter of the cylinder $b$. The exact wave resistance can be computed in closed form (see [12]) and is

$$
C_{w}=\frac{F_{x}}{\rho U_{\infty}^{2} b}=4 \pi^{2}(b / f)^{3} F r^{-6} \mathrm{e}^{-2 / F r^{2}},
$$

where the Froude number is defined as $F r=U_{\infty} / \sqrt{g f}$. For the partially submerged bodies the curve exhibits a series of oscillations due to interference of the wave patterns produced by the bow and the stern, specially at low Froude numbers as is the case for a hover-craft problem with a parabolic pressure variation on the surface, as shown in Fig. 7. The analytic expression for the wave resistance coefficient is

$$
C_{w}=\frac{F_{x}}{\rho U_{\infty}^{2} a}=16 \frac{(K a \cos K a-\sin K a)^{2}}{(K a)^{3}} .
$$

As in this case the support of the forcing term is compact, we can check for the invariance of the solution with respect to the position of the outlet boundary. We selected $a=1$ so that the forcing term is non-null in the range $|x|<1$, and we consider two equal meshes with $\Delta x=0.1$. One of them ends at $x=2$ while the other is extended to $x=6$, keeping $\Delta x=$ cnst. The wave profile for both meshes at $F r=0.8$ is shown superimposed in Fig. 8. They coincide to machine precision, as expected.

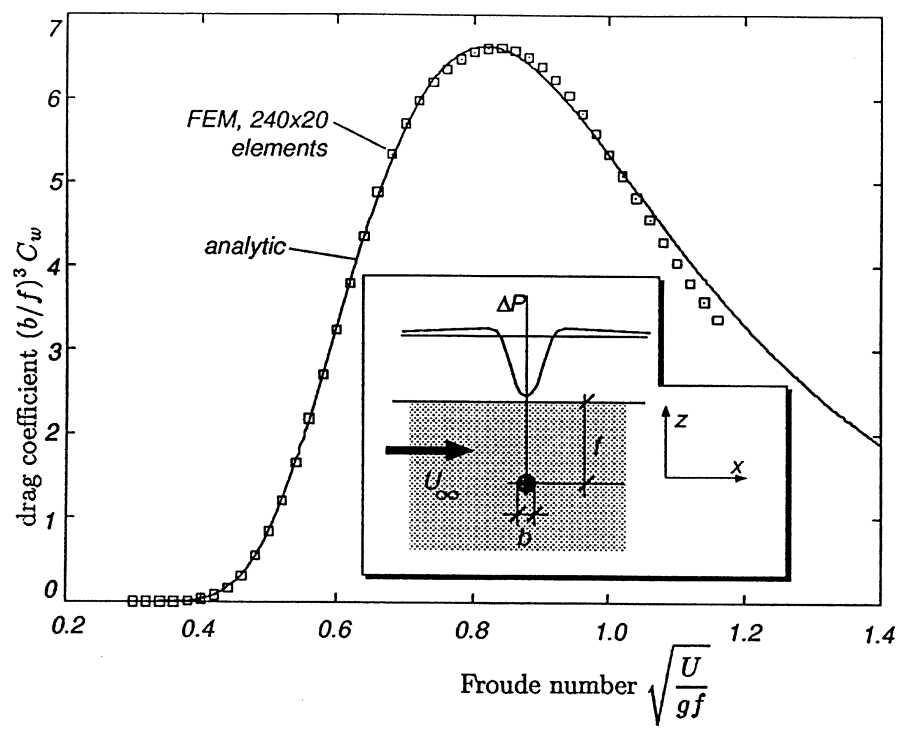

Fig. 6. Drag curve for the submerged dipole (cylinder with vanishing diameter). 


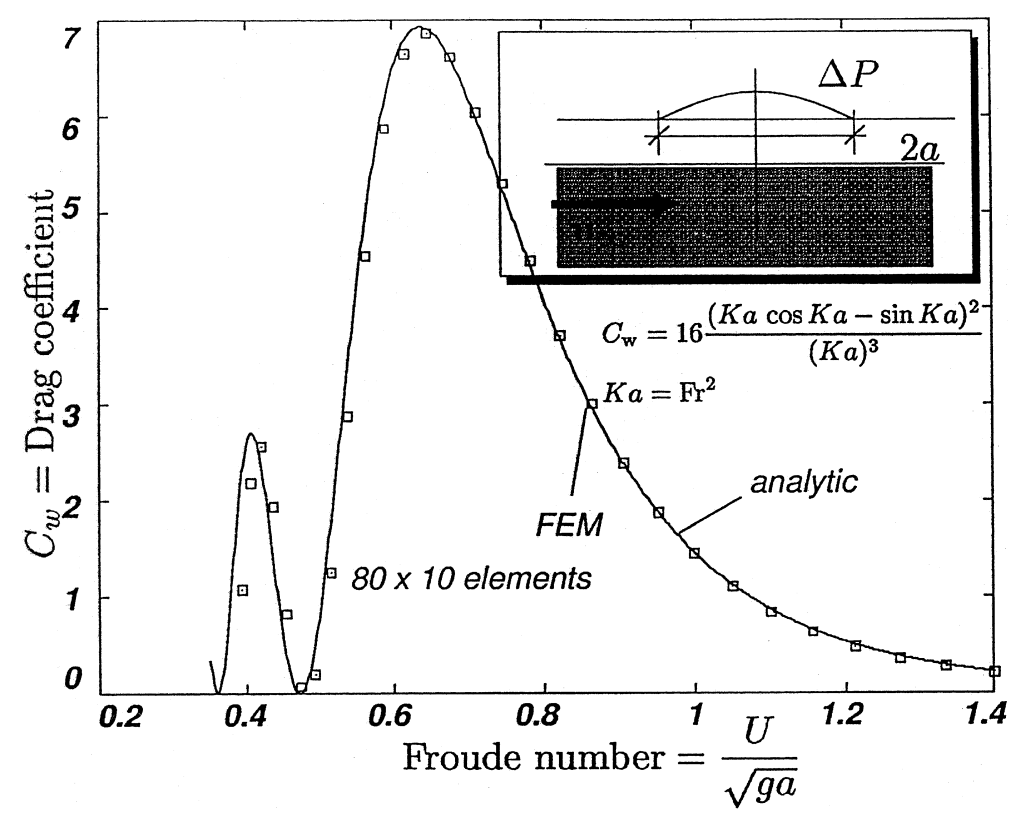

Fig. 7. Drag curve for the parabolic pressure distribution.

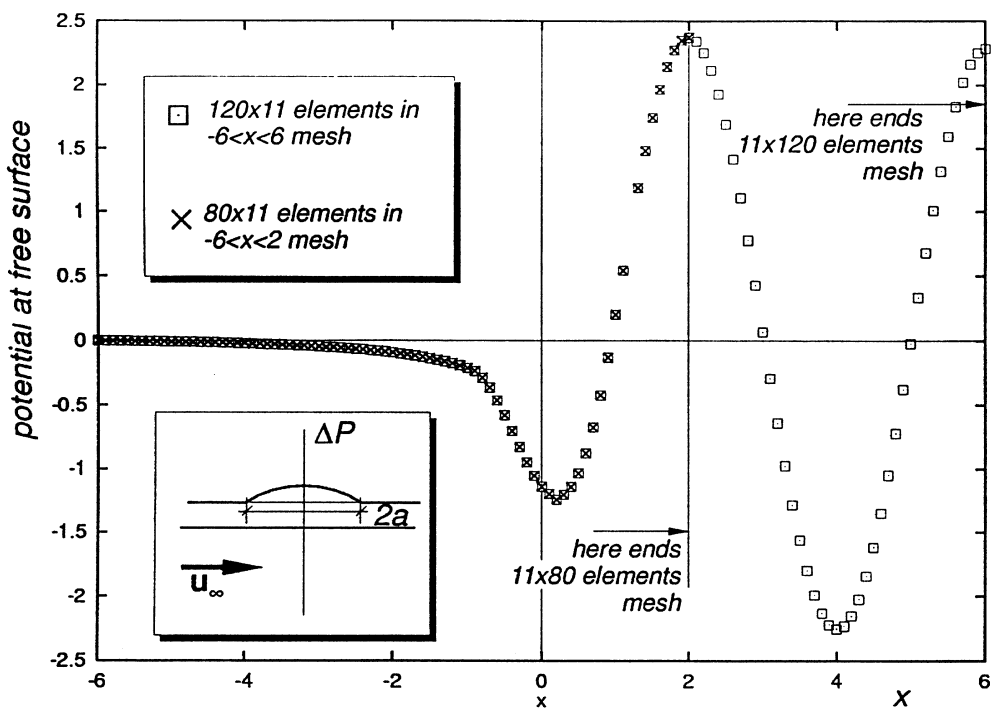

Fig. 8. Invariance of the discrete solution with the position of the absorbing boundary condition.

Results for, a 3D hover-craft problem with a rectangular pressure distribution for which experimental and analytical results are reported by Wehausen [18] is shown in Fig. 9 (the relation $L: B=3: 2$ is assumed). Finally, results for the well known Wigley hull (model $1805 \mathrm{~A}$ ) are shown in Fig. 10. The hull is defined by $y= \pm\left(1-x^{2} / 64\right)\left(1-0.6 x^{2} / 64\right)\left(1-z^{2}\right)$ for $|x|<8, z>-1$, and in this last case the drag coefficient is defined as (also known as circular $C$ coefficient)

$$
C_{w}=\frac{250}{\pi} \frac{F_{x}}{\Omega_{\text {ship }}^{2 / 3} \rho U_{\infty}^{2}},
$$

where $\Omega_{\text {ship }}=4(52 / 75)$ is the volume of the Wigley hull. 


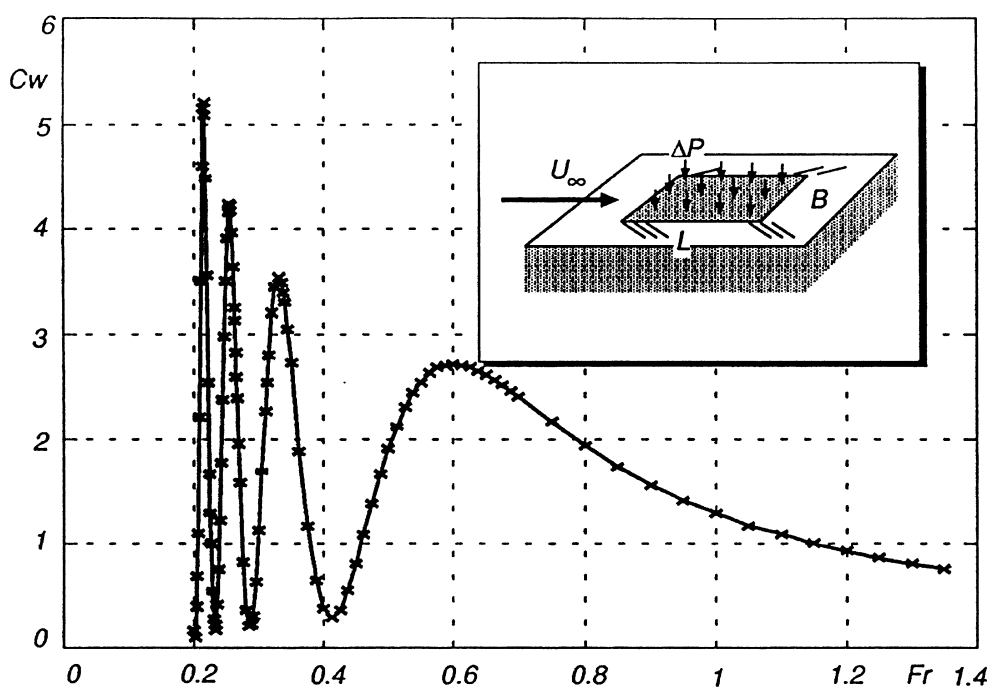

Fig. 9. Drag curve for the rectangular pressure distribution.

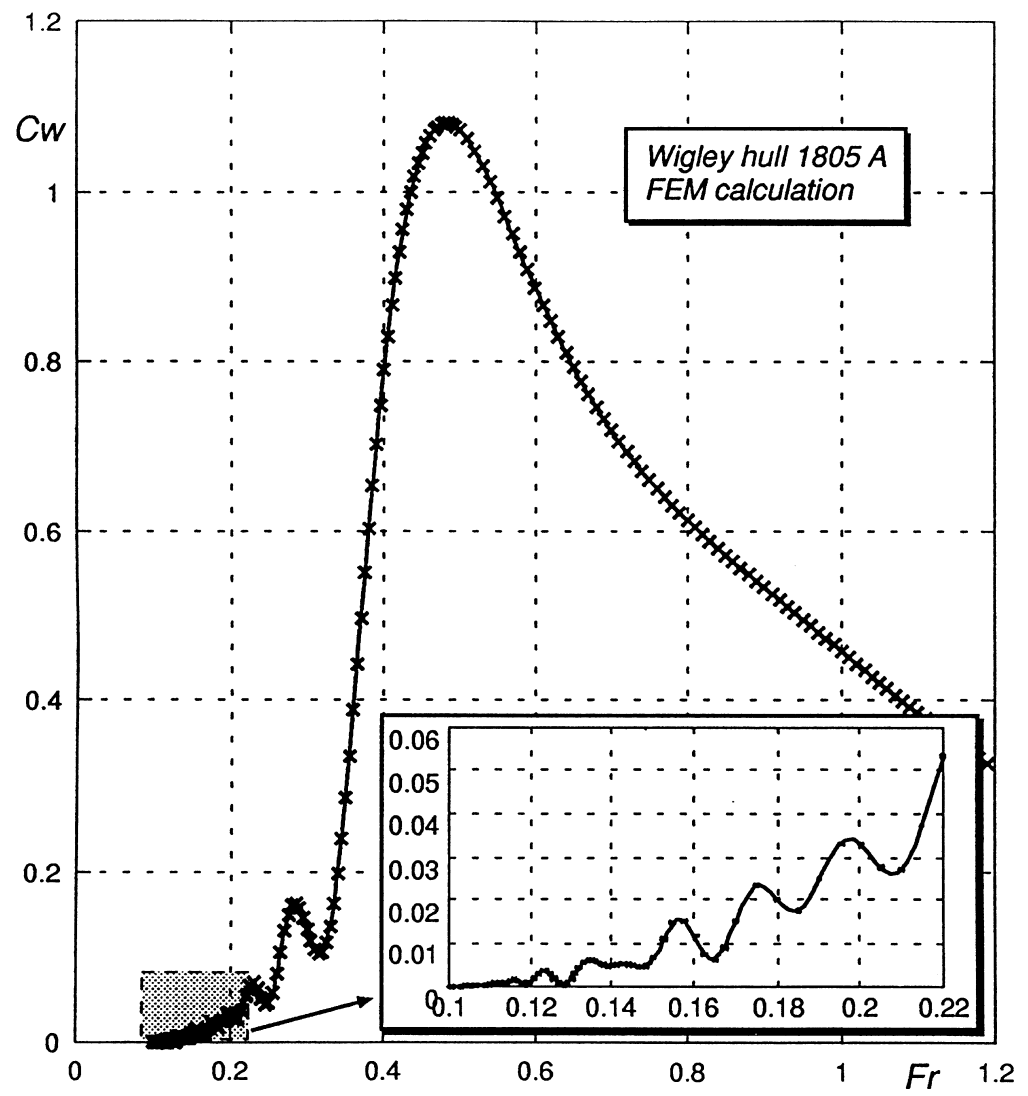

Fig. 10. Drag curve for the Wigley hull.

As can be seen from these numerical examples, drag curves computed with this method exhibit very well defined secondary maxima, and computations can be carried out for a wide range of Froude numbers. In addition, as no numerical viscosity is used, the wave-resistance can be computed from a momentum flux balance and positive wave resistances are guaranteed [17]. 


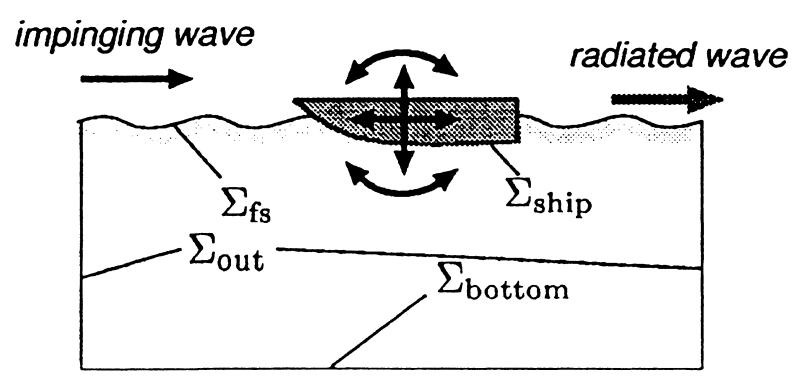

Fig. 11. Geometry description of the sea-keeping problem.

\subsection{The sea-keeping problem}

Sea-keeping refers to performance and safety of ships at rough seas $[15,14]$. The typical problem considered here is a plane wave impinging on a floating body or marine structure see Fig. 11. The body oscillates about its mean position due to these forces, and this movement originates in turn a pattern of radiated waves, resulting in a fluid structure interaction problem. From the numerical point of view, the problem amounts to solving (in 3D) the diffraction problem were the plane wave impinges on the body which is kept fixed, and a certain number of radiation problems were the impinging wave is not present, and the pattern of radiated waves for a certain prescribed harmonic motion of the body is computed. The number of radiation problems to be solved is equal to the number of degrees of freedom of the structure: three (two translations, one rotation) in 2D and six (three translations, three rotations) in 3D. The coupling of the fluid and the structure is made by solving a small system involving response coefficients computed for the fluid and the dynamical coefficients of the structure itself (mass, inertia moments, stiffnesses). We concentrate here in the solution of each of the fluid problems, either radiation or diffraction. The governing equations are

$$
\begin{aligned}
& \Delta \phi_{j}=0, \quad \text { in } \Omega, \\
& \phi_{j, n}-\left(\omega^{2} / g\right) \phi_{j}=0 \text { at } \Sigma_{\mathrm{fs}}, \\
& \phi_{j, n}=\left(\mathbf{u}-\mathbf{u}_{0}\right)_{j} \cdot \mathbf{n} \text { at } \Sigma_{\text {ship }}, \\
& \phi_{j}=0 \text { or } \phi_{j, n}=0 \text { at } \Sigma_{\text {bot }},
\end{aligned}
$$

appropriated radiation b.c.'s at $\Sigma_{\text {out }}$.

Condition (49) is the linearized free surface boundary condition, and can be obtained from elimination of the elevation from the linearized kinematic and dynamic conditions. (50) is the perturbation imposed either by the movement of the body or imposed by the impinging wave. Application of the DNL is straightforward and follows the lines described for the wave resistance problem discussed previously but with the difference that here the inviscid modes propagate in both directions, so that it results to be more similar to the Helmholtz equation. The problem is generally in cylindrical coordinates as shown in Fig. 12 , and three structured layers of nodes at the outer boundary serve for the computation of the DNL absorbing matrix.

The example here is a pile of varying section oscillating in heave mode (i.e. vertically) at a frequency such that the characteristic wave-length of the waves is $\lambda=2 \pi \mathrm{g} / \omega^{2}=0.7$, and the mean radius of the pile is $R=1$. In Figs. 13 and 14 we see the absolute value of the potential at the surface with and without absorbing boundary conditions, respectively. We see that with absorbing boundary condition, the result is almost independent of the position of the artificial boundary. Results are shown with two meshes, one ending at $r_{\text {out }}=3$ and the other with $r_{\text {out }}=5$. Note that in the former case the outer boundary is at two or three wave-lengths from the structure. In contrast, very small variations in the position of the outer boundary (from $r_{\text {out }}=10$ to $r_{\text {out }}=9.5$ ) cause large variations in the amplitude of the potential curve when no absorbing boundary conditions (i.e. a Neumann b.c.) are used, even when the position of the outer boundary is located relatively far from the body (more than 10 wavelengths). 


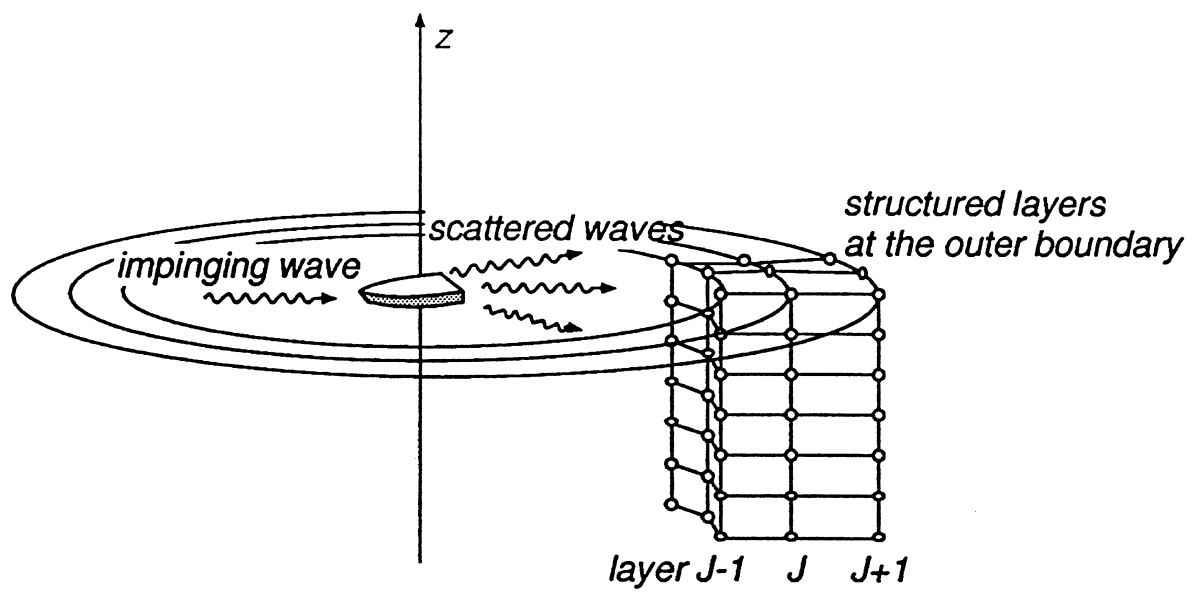

Fig. 12. Structured layers near the outlet cylindrical section for the computation of the DNL absorption matrix.

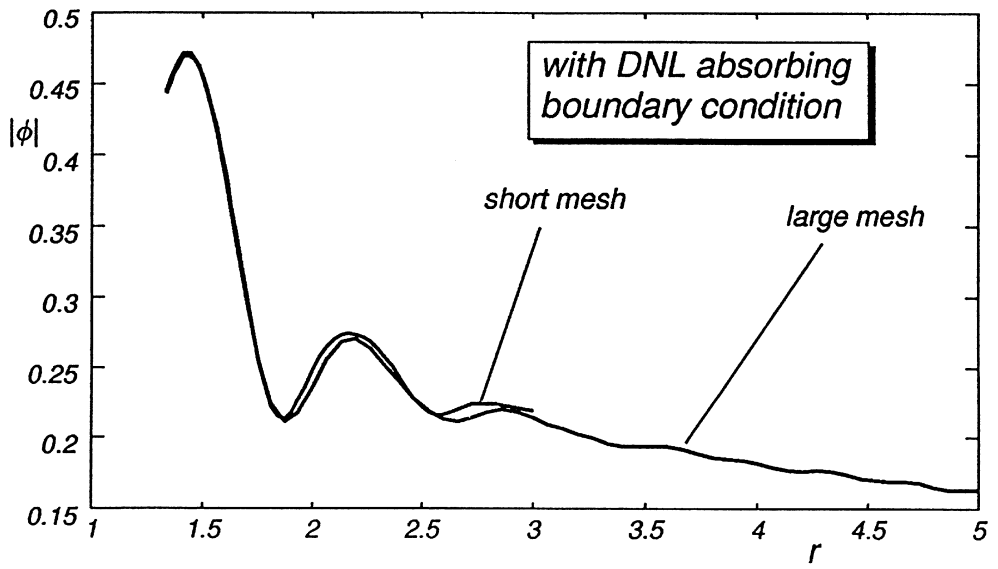

Fig. 13. Free surface elevation with DNL absorbing boundary conditions and two meshes of different external radii.

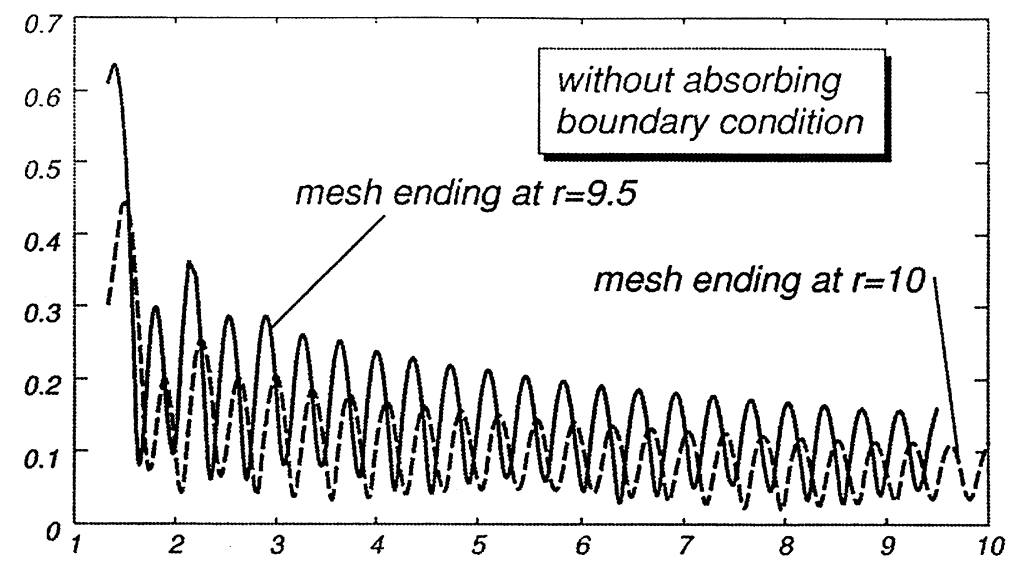

Fig. 14. Free surface elevation without absorbing boundary conditions and two meshes of different external radii. 


\section{Conclusions}

A discrete non-local (DNL) absorbing boundary condition for the wave-like problems has been presented. It is based on an eigen-decomposition of the system of ODE's that results from discretization in a $1 \mathrm{D}$ structured mesh. The sense of the propagation of the eigen-modes is determined depending on the sense of decay of the corresponding solution, that is on whether the absolute value of the corresponding eigenvalue is greater or lower than unity. For eigenvalues with unit absolute value the classification is performed by perturbing the operator with a small dissipative term, and it is shown that this is equivalent to computing the group velocity in the temporal form of the equation. Application, with numerical examples, to the Helmholtz equation, wave-resistance and sea-keeping problems is discussed.

\section{Acknowledgements}

This work has received financial support from Consejo Nacional de Investigaciones Científicas y Técnicas (CONICET, Argentina), Banco Interamericano de Desarrollo (BID) and Universidad Nacional del Litoral through grants BID/CONICET 802/OC-AR Pid Nr. 26, CONICET PEI 232/97 and CAI+D UNL 94/95. The work was done in cooperation with Centro Internacional de Métodos Numéricos en Ingeniería (CIMNE, Barcelona). We made extensive use of freely distributed software as Linux OS, Octave, Fortran $f 2 c$ compiler, Tgif and many others. We acknowledge a free single-user license for MuPAD from SciFace Software.

\section{References}

[1] J.C. Berkhoff, N. Book, A.C. Radder, Verification of numerical wave propagation models for simple harmonic linear water waves, Coastal Engrg. 6 (3) (1981) 255-279.

[2] P. Bettess, O.C. Zienkiewicz, Diffraction and refraction of surface waves using finite and infinite elements, Internat. J. Numer. Meth. Engrg. 11 (1977) 1271-1290.

[3] R.P. Bonet, N. Nigro, M.A. Storti, S.R. Idelsohn, Non-reflective planar boundary condition based on gauss filtering advances, In preparation 1998.

[4] J. Broeze, J.E. Romate, Absorbing boundary conditions for free surface wave simulations with a panel method, J. Comput. Phys. 99 (1992) 146.

[5] D.S. Burnett, R.L. Holfrod, Prolate and oblate spheroidal acoustic infinite elements, Comput. Methods Appl. Mech. Engrg. 158 (1998) 117-142.

[6] C.W. Dawson, A practical computer method for solving ship-wave problems, in: Proceedings of the second International Conference on Numerical Ships Hydrodynamics 30 Berkeley, 1977.

[7] J. D'Elía, Numerical methods for the Ship Wave-Resistance Problem, Ph.D. thesis, Univ. Nacional del Litoral (Santa Fe, Argentina) 1997

[8] D. Givoli, Non-reflecting boundary conditions, J. Comput. Phys. 94 (1991) 1-29.

[9] D. Givoli, J.B. Keller, A finite element method for large domains, Comput. Methods Appl. Mech. Engrg. 76 (1989) $41-66$.

[10] D. Givoli, J.B. Keller, Non-reflecting boundary conditions for elastic waves, Wave Motion 12 (1990) 261-279.

[11] Y. Ito, K. Tanimoto, A method of numerical analysis of wave propagation - application to wave diffraction and refraction, in: Proceedings of the 13th International Conference on Coastal Engineering 1972, ASCE, New York.

[12] L. Landweber, Motion of Immersed and Flotanting Bodies, Handbook of Fluid Dynamics, McGraw-Hill, New York, 1961.

[13] M. Lenoir, A. Tounsi, The localized finite element method and its application to the two-dimensional sea-keeping problem, SIAM J. Numer. Anal. 25 (1988) 729-752.

[14] J.N. Newman, Wave-drift of floating bodies, J. fluid Mech. 249 (1993) 241-259.

[15] M. Ohkusu, Advances in Marine Hydrodynamics, Computational Mechanics Publications, Wessex, 1996.

[16] M. Storti, J.D'Elía, S. Idelsohn, Algebraic discrete non-local (DNL) absorbing boundary condition for the ship wave resistance problem, to appear, 1998a.

[17] M. Storti, J.D’Elía, S. Idelsohn, Computating ship wave resistance from wave amplitude with the DNL absorbing boundary condition, 1998b.

[18] J.V. Wehausen, The wave resistance of ships, Advances Appl. Mech. 13 (1973) 93-245.

[19] O.C. Zienkiewicz, D.W. Kelly, P. Bettess, Marriage á la mode- or the best of both worlds. boundary integrals and finite element procedures, in: Proceedings of the Conference on Innovative Methods of Numerical Computation, Versailles, France, 1977. 